\title{
Assessment of the Fairness of Privacy Policies of Mobile Health Apps: Scale Development and Evaluation in Cancer Apps
}

Jaime Benjumea, MSc; Jorge Ropero, PhD; Octavio Rivera-Romero, PhD; Enrique Dorronzoro-Zubiete, PhD; Alejandro Carrasco, $\mathrm{PhD}$

Department of Electronic Technology, Universidad de Sevilla, Sevilla, Spain

Corresponding Author:

Jaime Benjumea, MSc

Department of Electronic Technology

Universidad de Sevilla

Escuela Técnica Superior de Ingeniería Informática

Avda Reina Mercedes s/n

Sevilla

Spain

Phone: 34630629719

Email: jaimebm@us.es

\section{Abstract}

Background: Cancer patients are increasingly using mobile health (mHealth) apps to take control of their health. Many studies have explored their efficiency, content, usability, and adherence; however, these apps have created a new set of privacy challenges, as they store personal and sensitive data.

Objective: The purpose of this study was to refine and evaluate a scale based on the General Data Protection Regulation and assess the fairness of privacy policies of mHealth apps.

Methods: Based on the experience gained from our previous work, we redefined some of the items and scores of our privacy scale. Using the new version of our scale, we conducted a case study in which we analyzed the privacy policies of cancer Android apps. A systematic search of cancer mobile apps was performed in the Spanish version of the Google Play website.

Results: The redefinition of certain items reduced discrepancies between reviewers. Thus, use of the scale was made easier, not only for the reviewers but also for any other potential users of our scale. Assessment of the privacy policies revealed that $29 \%(9 / 31)$ of the apps included in the study did not have a privacy policy, 32\% (10/31) had a score over 50 out of a maximum of 100 points, and $39 \%$ (12/31) scored fewer than 50 points.

Conclusions: In this paper, we present a scale for the assessment of mHealth apps that is an improved version of our previous scale with adjusted scores. The results showed a lack of fairness in the mHealth app privacy policies that we examined, and the scale provides developers with a tool to evaluate their privacy policies.

(JMIR Mhealth Uhealth 2020;8(7):e17134) doi: $\underline{10.2196 / 17134}$

\section{KEYWORDS}

privacy; mhealth apps; fairness assessment scale; cancer apps; GDPR

\section{Introduction}

\section{Privacy in Mobile Health Apps}

Health care systems are putting a great emphasis on the role of the patient and encouraging people to take control of their health [1]. Mobile health (mHealth) apps are one of the technological breakthroughs that make this possible. There are more than 3 billion smartphone users worldwide, and this number is predicted to grow by several 100 million in the next few years [2]. This proliferation of smartphones has led to an increase in the availability and abundance of mHealth apps. In 2017, there were more than $300,000 \mathrm{mHealth}$ apps, and this number tends to grow by $25 \%$ every year. In $2018,52 \%$ of smartphone users collected health-related information on their smartphones, and $60 \%$ of smartphone users downloaded health-related apps [3].

Among other uses, mHealth apps can provide disease and treatment information; practical tools for avoiding some diseases (prevention and healthy behavior promotion); tools to assist in the identification of symptoms (early detection); practical tools to deal with the medical, behavioral, or emotional aspects of a 
specific disease (disease management); and access to peer or professional assistance (support) [4,5].

Despite the potential impact of mHealth apps on patient health, there is a lack of specific regulations and standards regarding the development of mHealth apps [6], which may result in potential risks and poor mHealth app quality. For example, some studies have reported problems with existing mHealth apps such as failure to meet the needs of persons with chronic conditions [7], a lack of cited source material or references [5], and insufficient testing of mHealth apps with respect to usability and validity [8]. Such setbacks reduce health care professional and patient confidence in these apps $[9,10]$.

Criteria have been proposed to assess mHealth apps. Stoyanov et al [11] developed the Mobile Application Rating Scale (MARS) scale to classify and rate the quality of mHealth apps based on a literature review of app evaluations containing explicit quality rating criteria. Llorens-Vernet and Miró [6] recently proposed criteria to be integrated into a general standard for mHealth app development based on a systematic review, searches on professional organization websites, and standards governing the development of software for medical devices.

Privacy is a major concern for mHealth app users [12], as some mHealth apps require the collection, storage, and sharing of personal and sensitive patient data. Guidelines and recommendations for health-related apps-such as those developed by the Andalusian Agency for Healthcare Quality (Spain), Tecnologies de la Informació i la Comunicació Salut Social Foundation (Spain), National Health Service (United Kingdom), and European Commission-include several privacy items that highlight its importance in the context of mHealth. Also, privacy is one of the components included in the criteria proposed by Stoyanov et al [11] and by Llorens-Vernet and Miró [6].

Privacy assessment is a multifaceted issue that, among other things, bears upon privacy policies. In the context of mHealth apps, privacy policies contain privacy-related information that users can review prior to installation in order to get a clear idea of what personal data the app will access and the purposes for their processing. These concerns should be taken seriously, but $70 \%$ of the 600 most-used health-related apps do not include a privacy policy [13]. Although the previously mentioned guidelines and recommendations, MARS scale, and criteria include items regarding privacy, developers and evaluators need more specialized tools when it comes to the development and assessment of mHealth app privacy policies.

In this paper, we introduce a novel scale based on the General Data Protection Regulation (GDPR) to assess the fairness of mHealth app privacy policies. This scale provides detailed information regarding items to be included in privacy policies in order to comply with the GDPR. Consequently, we offer an objective and reproducible method for assessing the fairness of mHealth app privacy policies or developing the privacy policy of an mHealth app. This paper presents the final version of the scale (the culmination of an iterative development process) and the results obtained after applying it in a case study.

\section{Legal Background}

The GDPR is a regulation (2016/79) passed by the European Parliament and the Council of the European Union (EU). It was published in the Official Journal of the European Union [14] in 2016 and has been applicable since May 25, 2018. The GDPR applies to all EU member countries plus Iceland, Luxemburg, and Norway. Being a regulation (and not a directive), the GDPR applies directly to all these countries.

The GDPR introduces some important changes that replace previous legislation (Directive 95/46/EC). The first one can be found in Article 3 (territorial scope), as the GDPR applies to any controller or processor in the EU, even if processing does not take place in the EU. Also, the GDPR applies to any controller or processor (regardless the country of origin) if it is related to "the offering of goods or services, irrespective of whether a payment of the data subject is required, to such data subjects in the Union or the monitoring of their behavior as far as their behavior takes place within the Union" (Article 3.2). The controller must be able to demonstrate compliance with the GDPR (Article 5.2) and is subject to higher fines than before (Articles 66 and 83). Article 4 of the GDPR includes definitions that clarify relevant concepts. These concepts are summarized in Table 1.

Like many legal texts, the GDPR is difficult to understand and comply with, especially when it comes to app developers or users who are not legal experts; reading, interpreting, and understanding 99 articles in 88 pages of legal language is not easy. Our paper helps ordinary people become more familiar with the regulations so they can comply with the laws. We have developed a tool that makes compliance as easy as following simple guidelines such that even small app developers (eg, freelancers) can easily use it.

For example, there are two items in our scale (items 4 and 5) regarding the information to be provided to the data subject, described in Article 13 with a simple sentence: "the purposes of the processing for which the personal data are intended as well as the legal basis for the processing." This sentence is translated in the definition of our scale into approximately 250 words because this article is connected with several parts of the GDPR: recitals 39, 58, 60, 61, and 63, and articles 4, 5, and 6 . These recitals and articles must be read and understood to be clear on the intentions of the GDPR. 
Table 1. Definition of General Data Protection Regulation concepts.

\begin{tabular}{|c|c|}
\hline Concept & Definition \\
\hline Data subject & $\begin{array}{l}\text { A natural person whose personal data are being processed; the GDPR }{ }^{\mathrm{a}} \text { defines personal data not only as the data related } \\
\text { to an identified person, but also as the data that can be used to identify, directly or indirectly, a natural person. }\end{array}$ \\
\hline Data controller & $\begin{array}{l}\text { "The natural or legal person, public authority, agency, or other body which, alone or jointly with others, determines the } \\
\text { purposes and means of the processing of personal data" [14]. }\end{array}$ \\
\hline Data processor & $\begin{array}{l}\text { "The natural or legal person, public authority, agency, or other body which processes personal data on behalf of the con- } \\
\text { troller" [14]. }\end{array}$ \\
\hline Recipient & "The natural or legal person, public authority, agency, or another body, to which the personal data are disclosed" [14]. \\
\hline Representative & $\begin{array}{l}\text { A natural or legal person established in the } \mathrm{EU}^{\mathrm{b}} \text {; a representative must be designated by data controllers or processors not } \\
\text { in the EU (Article 27). }\end{array}$ \\
\hline $\mathrm{DPO}^{\mathrm{c}}$ & $\begin{array}{l}\text { A person who must be designated by the controller or processor in certain circumstances (see Article } 37 \text { for more details); } \\
\text { the duties of the } \mathrm{DPO}^{\mathrm{c}} \text { are defined in Article } 39 \text {; they include, among others, advising the controller or processor about } \\
\text { their duties related to the GDPR and monitoring compliance with GDPR }\end{array}$ \\
\hline
\end{tabular}

${ }^{\mathrm{a}}$ GDPR: General Data Protection Regulation.

${ }^{b}$ EU: European Union.

${ }^{\mathrm{c}}$ DPO: data protection officer.

\section{Research Background}

There are several studies in the literature that have addressed the availability of privacy policies in mobile apps and the assessment of some aspects of their quality [9,15-37].

Heuristics were proposed by Hutton et al [15] to assess privacy in mHealth apps for self-tracking. In their study, Hutton et al [15] used recommendations from the US Federal Trade Commission and GDPR to define the items used in the heuristics. The evaluation of an app consists of checking its behavior and the contents of its privacy policy. They also proposed a scoring method. However, their system is not exclusively based on the GDPR and evaluates other issues that are not related to the content of the privacy policy. For example, some items in the heuristics are closely related to usability.

In 2013, Sunyaev et al [16] assessed the availability and quality of the privacy policies of the 600 most commonly used mHealth apps. They found that only 183 apps out of 600 had a privacy policy. They also determined the lengths of privacy policies, their readability, and whether privacy policies were focused on the app. Moreover, they checked if the contents of privacy policies addressed aspects users considered to be the most important. They did not develop a method or use any legal framework or regulation to design their assessment scheme.

A framework for assessing apps related to chronic insomnia disorder was defined by Leigh et al [17]. The framework was based on 24 criteria, and 6 of them dealt with privacy policies. The framework defines 6 questions about the content of the privacy policy that must be answered yes or no based on the UK Data Protection Act 1998 (and thus, Directive 95/46/EC), the UK Information Commissioner Office, and the Charter of Fundamental Rights of the EU. The authors used the answers to these 6 questions to obtain a privacy score. Blood pressure and diabetes apps were assessed by Knorr et al [18] in a study that rated 154 apps using static and dynamic analysis and evaluating web server connections and privacy policies. They assessed 12 aspects of the privacy policies including recommendations from the Organization for Economic Cooperation and Development on privacy. They discovered that $67 \%$ of the apps that stored data on the web had a privacy policy. However, they neither developed a score to evaluate privacy policies nor did they compare them.

A scoring method to evaluate the quality of 116 apps for depression was defined by O'Loughlin et al [19] using 7 questions about the privacy policy and app behavior, such as whether the app requires a personal identification number for access or the privacy policy states if data are encrypted or stored locally. Based on the results of this questionnaire, they classified privacy policies as acceptable, questionable, or unacceptable, but they did not assign a numerical score to the app, and it is not GDPR-based. They also found that only 57 out of the 116 apps they evaluated had a privacy policy.

Huckvale et al [9] assessed 79 mHealth apps, certified as clinically safe and trustworthy by the United Kingdom National Health Service Health Apps Library. They evaluated the app behavior, considering aspects like data transmission, storage, and privacy policies. When assessing privacy policies, they used a coding method based on the UK Information Commissioner Office recommendations and the UK Data Protection Act. Their proposed scheme used four domains (uses of data, technical concerns, user rights, and administrative details) to classify the topics analyzed in the privacy policy. Each of the 24 topics was classified as addressed or absent in the privacy policy. Although they discussed the percentage of apps complying with each topic, they did not develop a scoring method.

Papageorgiou et al [20] conducted a privacy and security analysis of $20 \mathrm{mHealth}$ apps. They had a broad scope of analysis including static and dynamic analysis of the apps, permission analysis, and security in communications. They also investigated whether privacy policies complied with the GDPR, focusing on the right to withdraw consent, the right to portability, data protection officer (DPO) contact information, profiling, and transfers of personal data to countries not within the EU. They 
discussed how many apps complied with these items, but they also did not develop a scoring method.

A total of 29 apps were analyzed by Minen et al [21], focusing on data storage and privacy policies in headache apps. When analyzing privacy policies, they searched for the presence or absence of information regarding data collection, data sharing, use by children, and certain user rights.

The scientific community has been searching for a way to assess privacy in mHealth apps for the last few years. Many studies have assessed privacy in apps, usually focusing on their user interfaces, privacy in communications, and privacy policies. However, the established criteria used to analyze privacy policies are heterogeneous and subjective. These solutions are based on the researchers' own experience, the literature, and/or an existing legal framework. The items that are considered for the assessments are very diverse, and the evaluation of these items are, on many occasions, very subjective to the evaluators' criteria.

It is necessary, therefore, to create tools to evaluate privacy policies and establish privacy scales according to objective criteria that are less open to interpretation. Although some papers considered the GDPR, none of them proposed a set of items that enable GDPR compliance. Our aim is to fill that research gap by proposing of a GDPR-based scale to assess privacy policies in mHealth apps.

\section{Methods}

\section{Privacy Scale Design}

Article 13 of the GDPR summarizes the information that must be given to the user (known as data subject in the GDPR) when the information is collected from them (Table 1). This information is usually delivered to the user via a document called a privacy policy, and in order to be compliant with the GDPR, the privacy policy must meet certain requirements.

In our previous work [38], we developed a scale to assess the fairness of the privacy policies of mHealth apps. The objective of our scale was to analyze privacy policies in a systematic way and design a GDPR-based system to assess and improve such policies. Based on Article 13 of the GDPR and the recommendations of the National Data Protection Authority in Spain $[39,40]$, we identified and summarized the information that should be provided in privacy policies (see Table 2 for a list of the items). We also defined a scoring method to assess each item.

Our scale is not intended to check strict compliance with the GDPR but to assess the fairness of privacy policies. This means that a privacy policy with fewer than 100 points (the maximum score) may be compliant, in a strict way, with the GDPR. This is because some items mentioned in Article 13 (for example, the identity of the data controller) must be analyzed carefully. It is not always easy to classify an item as yes or no in terms of compliance with the GDPR. Also, the GDPR allows some items to be omitted in certain cases, such as transfer to non-EU countries if the personal data are stored in the EU. However, our proposed score penalizes the absence of such information even if the data remain in the EU. In fact, we are just applying one of the principles of the GDPR (Article 5)- "lawfulness, fairness, and transparency" - to privacy policies, as they should go slightly further in their contents.

Indeed, the items defined in our scale may be used by developers as a checklist to design privacy policies that comply with the GDPR. They also could be used by data controllers to check if their apps are GDPR-compliant. Furthermore, a privacy policy scoring 100 points would be fully compliant with the GDPR. Using the proposed scoring method, we consider privacy policies with scores from 75.0 to 100 points as very fair (category 1 [Cat1]). A score from 50.0 to 74.9 is somewhat fair (category 2 [Cat2]). A score from 25.0 to 49.9 is somewhat unfair (category 3 [Cat3]). Finally, we consider a score from 0 to 24.9 as very unfair (category 4 [Cat4]).

Some discrepancies in the interpretation of how to assign a score to some items were found in the first iteration of the scale design process. In the first iteration, the privacy policies of 9 apps were analyzed, and we obtained Kappa-Cohen indexes for each item. Possible scores for each item and Kappa-Cohen indexes are shown in Table 3. 
Table 2. Items in the privacy policy (Article 13).

\begin{tabular}{ll}
\hline Item & Item number \\
\hline Identity of data controller & 1 \\
Identity of the representative & 2 \\
Data protection officer details & 3 \\
Purposes for the processing & 4 \\
Legal basis for the processing & 5 \\
Legitimate interests from controller & 6 \\
Recipients (or categories) of the personal data & 7 \\
Transfers to non-European Union countries & 8 \\
Period for which data will be stored & 9 \\
Existence of data subject's rights & 10 \\
Existence of right to withdraw consent & 11 \\
Right to lodge a complaint with a supervisory authority & 12 \\
Obligation to provide personal data & 13 \\
Existence of automated decision making or profiling & 14 \\
\hline
\end{tabular}

Table 3. Kappa-Cohen indexes for privacy policy items for the 9 apps evaluated in the first iteration.

\begin{tabular}{|c|c|c|c|}
\hline Item & $\begin{array}{l}\text { Item } \\
\text { number }\end{array}$ & Score & $\begin{array}{l}\text { Kappa-Cohen in- } \\
\operatorname{dex}(\mathrm{n}=9)\end{array}$ \\
\hline Identity of data controller & 1 & 0: no info; 0.5 : partial; 1 : full & 0.77 \\
\hline Identity of the representative & 2 & 0: no info; 1 : info provided; N/A: not applicable & 1 \\
\hline Data protection officer details & 3 & 0 : no info; 1 : info provided & 0.61 \\
\hline Purposes for the processing & 4 & 0: no info; 0.5 : generic; 1 : specific & 0.77 \\
\hline Legal basis for the processing & 5 & 0: no info; 1 : info provided & 0.77 \\
\hline Legitimate interests from controller & 6 & 0: no info; 1 : info provided; N/A: not applicable & 0.8 \\
\hline Recipients (or categories) of the personal data & 7 & 0 : no info; 1 : info provided & -0.13 \\
\hline International transfers of data & 8 & $\begin{array}{l}0: \text { no info; } 0.5 \text { : generic; } 1 \text { : full details or no international } \\
\text { transfers }\end{array}$ & 0.53 \\
\hline Period for which data will be stored & 9 & 0: no info; 0.5 : generic; 1 : specific & 0.66 \\
\hline Existence of data subject's rights & 10 & 0 : no info; 0.5 : generic; 1 : full & 0.49 \\
\hline Existence of right to withdraw consent & 11 & 0: no info; 1 : info provided; N/A: not applicable & 0.08 \\
\hline Right to lodge a complaint with a supervisory authority & 12 & 0: no info; 0.5 : generic; 1 : specific & 0.77 \\
\hline Obligation to provide personal data & 13 & 0: no info; 1 : info provided & -0.17 \\
\hline Existence of automated decision making or profiling & 14 & $\begin{array}{l}0: \text { no info; } 0.5 \text { : generic; } 1 \text { : specific or no profiling or auto- } \\
\text { mated decision making done }\end{array}$ & 0.17 \\
\hline
\end{tabular}

A refinement of the criteria used to assign those scores was performed to resolve the discrepancies. Also, an error in the definition and description of one of the items was corrected. Items and their possible scores are (re)defined as follows:

- Identity of data controller: 1 point if full information is given. Full information means name, postal address, and electronic address (both email and a contact form are considered valid) of the data controller; 0.5 points if some information is missing; 0 points if the information is omitted. If only an electronic address is provided, the score is 0 points. Also, if the street address is not mentioned, the score is 0 points.

- Identity of the representative: The representative is a natural or legal person, established in the EU, who must be designated by the data controller if they are not in the EU. In this case, 1 point is given if full information is given (in the same way as with the data controller) and 0 points otherwise.

- DPO details: The GDPR states that a DPO must be designated if the controller processes a large quantity of data in some special categories, such as health data. We assume that a DPO must exist in any given mHealth app. 
At least an email address must be given to get 1 point. In order to be consistent with the definition of DPO in the GDPR, the DPO must be a different person from the data controller, so the email address should also be different. Otherwise, 0 points are given.

- $\quad$ Purposes for the processing: The purposes for the processing must be stated explicitly in the privacy policy. Sometimes the information given is too general. For example, "We collect this information for the purpose of providing our service" does not give any detail about why the data controller needs the personal data. In this case, the score for this item is 0.5 points. If purposes are provided explicitly, 1 point is given. If purposes are not mentioned, 0 points.

- Legal basis for the processing: There are six legal bases for the processing (Article 6 of GDPR): consent, need to perform a contract, legal obligation, protect vital interest of somebody, public interest or exercise official authority, and legitimate interest. This information must be given in the privacy policy in order to get 1 point. However, we found that, in some cases, the legal basis in which the processing was founded was not explicitly stated in a separate paragraph. This is because, sometimes, this information is embedded in the declaration of the purposes for the processing. As the information is, after all, given to the data subject, we decided to give 1 point in these cases. Furthermore, if 0 points are given to this indicator, indicators based on the legal basis (ie, items 6 and 11) are considered N/A (not applicable).

- Legitimate interests from controller: 1 point if this information is given and 0 points otherwise. N/A if legitimate interest is not stated as a legal basis for the processing or if item 5 is 0 points.

- Recipients (or categories of recipients) of the personal data: 1 point if this information is given and 0 points otherwise. We must note that if there are no recipients, this must be explicitly stated. Also, we must keep in mind that in accordance with Article 4 of the GDPR a data processor is considered a recipient.

- Transfers to non-EU countries: This item refers to the fact that personal data may be transferred to a country not in the EU. In this case, the data controller must give enough information about the measures that are in place to achieve a similar level of protection. We give 1 point if privacy policy indicates that this transfer is in place, and there is a reference to the measures taken. We consider enough information to contain a mention to the compliance with Privacy Shield [14] or similar frameworks. We also give 1 point if the transfer is based on an adequacy decision from the Commission. If there is a mention to a transfer to non-EU countries without further information, this item is 0.5 points. The GDPR states that this information must be given if there are transfers to non-EU countries and says nothing if the data are stored within the EU. We consider that this information must be given even where there are no transfers to non-EU countries. Thus, if there is no information about transfers outside the EU, this item is 0 points. When the data controller is in the EU, the fact of not transferring data outside the EU must be explicitly stated. If not, the item is 0 points. Otherwise, the item is N/A.

- Period for which data will be stored: To obtain 1 point, the privacy policy must point out a specific time in the future when the data will be erased. We consider the following time references as valid: a period of inactivity in the data subject's account or a specific reference to a user request to erase the data. The latter is independent of the specification of data subject's rights in the privacy policy.

- Existence of data subject's rights: 1 point is given if the specific user's rights mentioned in Article 13 (right to access, rectification, erasure, restriction of processing, object of processing, and data portability) are enumerated in the privacy policy with a way to exercise these rights. This information may also be provided using a link. We award 1 point if 5 or more rights are mentioned and 0.5 points if partial information is given (for example, some rights are omitted or there is no indication on how to exercise the rights); 0 points if there is no reference to data subject's rights.

- Existence of the right to withdraw consent: This is an additional right that only exists if the legal basis for the processing is consent. As such, the score is 1 point if this right is mentioned (along with the way to exercise it) and 0 points if the right is omitted. This item is N/A if consent is not one of the legal bases for the processing or if item 5 is 0 points.

- $\quad$ Right to lodge a complaint with a supervisory authority: According to the GDPR, there is an obligation to inform users that they have the right to lodge a complaint with a supervisory authority if they believe that their rights have been violated. To score 1 point, the privacy policy must not only identify the appropriate supervisory authority but also provide at least a link to it. Moreover, 1 point is given if the policy links to the list of all supervisory authorities within the EU. Simply naming the supervisory authority is considered insufficient and earns a score of 0.5 points and 0 points otherwise.

- Obligation to provide personal data: The privacy policy must explicitly state what happens if the user does not provide certain personal data. Examples of good practices are the following: "if you choose not to provide data, we may not be able to provide you those services" and "In order to join [...] you must provide [...]." If this information is given, 1 point. Otherwise, 0 points.

- Existence of automated decision making or profiling: We consider that there must be a reference to the existence or absence of automated decision making or profiling based on personal data. This item may be tricky to interpret because sometimes apps make automated decisions such as defining user interface language based on personal data. This example probably does not fit within the reasoning of the GDPR. Thus, we consider as automated decision making or profiling a behavior that goes beyond simple decisions made by the app. This item scores 1 point if this information is shown in the privacy policy and enough information about the logic around this decision or profiling is given. We consider a link to the information to be valid. Therefore, 0.5 points are scored if there is a reference to this item with 
no additional information, and the score is 0 points if no information is given. Simply using Google Analytics is not sufficient to consider that the app to be profiling. However, the use of cookies, if they modify the app behavior, is considered profiling. We are also aware that, in accordance with the National Data Protection Authority in Spain on mobile apps [41], some information must be given if the app includes targeted advertisements.

As stated in the introduction, an important objective of our research is to provide a reproducible scale for the assessment of privacy policies in mHealth apps, so schematics and further descriptions of the items with explanatory examples for the scoring system are defined in Multimedia Appendix 1.

The proposed scale consists of 14 items to check when assessing a privacy policy, as shown in Table 1 . Each item is assigned a score of 0 points or 1 point, though some of them may score 0.5 points. Thus, every item has the same weight within the total score. Also, as some items may be N/A, the final score is expressed as a percentual score. Thus, if an app achieves 7 points when privacy items are assessed but only 12 items are applicable, its final score is 58.3 points. Like the scales previously defined in the literature, our scale must be simple and easy to apply. Our scoring method is in concordance with other mHealth app privacy scales [15,27,32], which are also composed of yes/no questions. Most of the privacy scales that defined a score were based on items that could have 2 or 3 values.

Items $1,4,8,9,10,12$, and 14 can score 0.5 points, as some items are more complex than others. For example, the item purposes for the processing requires that the data controller be very explicit when defining the app's purposes for the processing. We found that some data controllers state the purposes for the processing but are not as explicit as they should be. In these cases, the item scores 0.5 points. Other items, such as item 3 (DPO details) are so simple that they can only score 1 point (yes) or 0 points (no). Additional details and examples can be found in Multimedia Appendix 1.

We have also added new indicators that did not appear in our previous work. They do not directly influence scores, but they add some information that is relevant to the study of privacy policies:

- Date of last update of the privacy policy: We look for this information in the text of the privacy policy itself. We do not consider alternative ways of obtaining the last update date such as analyzing http last-modified headers [42].

- Data controller's country: We collect this from the identity of the data controller. We do not consider additional information such as any obtained by the WHOIS tool [43]. This tool provides information about the domain name, but it might be misleading.

- GDPR awareness: This indicator only gets a yes/no value indicating whether GDPR is explicitly mentioned in the privacy policy.

\section{Case Study: Cancer Apps}

\section{Study Design}

A systematic search strategy was followed to identify all relevant mHealth apps for the most common types of cancer (breast, prostate, colorectal, and lung cancer) and for cancer in general. We focused on Android apps due to its market dominance, being the most installed operating system among the new smartphones shipped worldwide from 2017 to 2019 [44]. Two researchers (ORR and EDZ) searched the Spanish version of the Google Play website, taking steps to ensure that no previous searches or cookies influenced the results. Five searches were completed on July 25, 2019, using "cancer mama," "cancer prostata," "cancer," "cancer colon recto," and "cancer pulmon" as search strings.

\section{Selection Criteria}

Apps were included in the screening stage if their title or description contained one of the search strings defined. After duplicates were removed, two researchers (ORR and EDZ) reviewed and assessed the title and description of the resulting mHealth apps for eligibility against the selection criteria. Apps whose titles and/or descriptions met the selection criteria were downloaded and installed. A researcher (ORR) checked that they worked properly and met the selection criteria. Disagreements were resolved by consensus.

The following inclusion criteria were used: the title or description referred to at least one of the search strings, it was intended exclusively for cancer patients or survivors, and the app collected user data or allowed users to share their opinions or data.

Apps were excluded if they met at least one of the following conditions: the title or description was written neither in English nor Spanish, the user interface was available neither in English nor Spanish, the privacy policy was written neither in English nor Spanish, it was not focused on cancer, it was intended for people other than cancer patients or survivors, or it was not free.

\section{Data Extraction}

The following descriptive characteristics of apps meeting the selection criteria were collected from the Google Play website when available: developer, category, number of ratings, user rating, last update, and number of downloads. Additionally, using the information included in the description, two researchers (ORR and EDZ) independently classified the apps according to main purpose and type of cancer. Discrepancies were resolved by consensus.

URLs linking to the privacy policy of the included apps were also collected when available. If no link was provided, we tried to find the privacy policy on the developer's website. A researcher (JR) reviewed all installed apps and checked if they contained any additional privacy policy information. If the privacy policy contained in the app was different from the one linked in the Google Play website, the former was considered for the assessment. When user registration was required, the privacy policy had to be available before registering for the service. Otherwise, we considered the app to not have a privacy policy, as it was not accessible before using the app. Two 
researchers (JB and JR) reviewed and independently assessed all privacy policies of the included apps using the proposed scale. Finally, a score for each privacy policy was assigned according to the scoring scale defined earlier. Discrepancies were resolved by consensus.

\section{Classification}

Included apps were classified according to main purpose and type of cancer. We used the classification scheme for an app's main purpose proposed in Giunti et al [7]. We removed the awareness-raising option because the apps designed for this purpose did not meet the selection criteria and were excluded from our study. Therefore, we coded an app's main purpose as disease and treatment information (DTI), disease management (DM), or support (S).

Finally, type of cancer was coded as general, colorectal, breast, prostate, lung, or other. General was used to code included apps that pertained to cancer in general without identifying any specific type. Other was assigned to apps that pertained to a specific type of cancer other than colorectal, breast, prostate, or lung cancer.

\section{Results}

\section{App Selection and Extracted Features}

Google Play searches resulted in 1249 mHealth apps. After duplicates were removed, 831 mHealth apps were assessed for eligibility; 41 of those apps met the selection criteria and were downloaded and installed on an Android smartphone (Moto G7, Motorola Mobility, LLC) to check if they worked properly. Finally, $31 \mathrm{mHealth}$ apps met the selection criteria and were included in the analysis. Figure 1 shows the flow diagram of the described procedure, while Table 4 shows the selected apps considering the selection criteria. For convenience when analyzing the results, apps were tagged from App1 to App31. For the statistical analysis below, five more features were added to Table 4: Google Play app ratings, number of reviews, number of downloads, app type, and cancer type. Multimedia Appendix 2 contains a list of the apps that were found in Google Play search and those included in the case study.

We applied the second iteration of our privacy policy assessment scale (described in the Methods section) to the 31 selected apps. In the case of App15, a privacy policy link led to a generic privacy policy for the company, so we considered the one in the app. Table 5 shows the privacy scores obtained after assessing all the privacy policies. We also added more information about the apps to the table in order to achieve a more complete analysis of the results. In particular, we added the data controller's location, the last app update, and whether the privacy policy was GDPR-aware (ie, it mentioned the GDPR). If the app did not have a privacy policy, it was assigned a score of 0 points. 
Figure 1. Flow diagram.

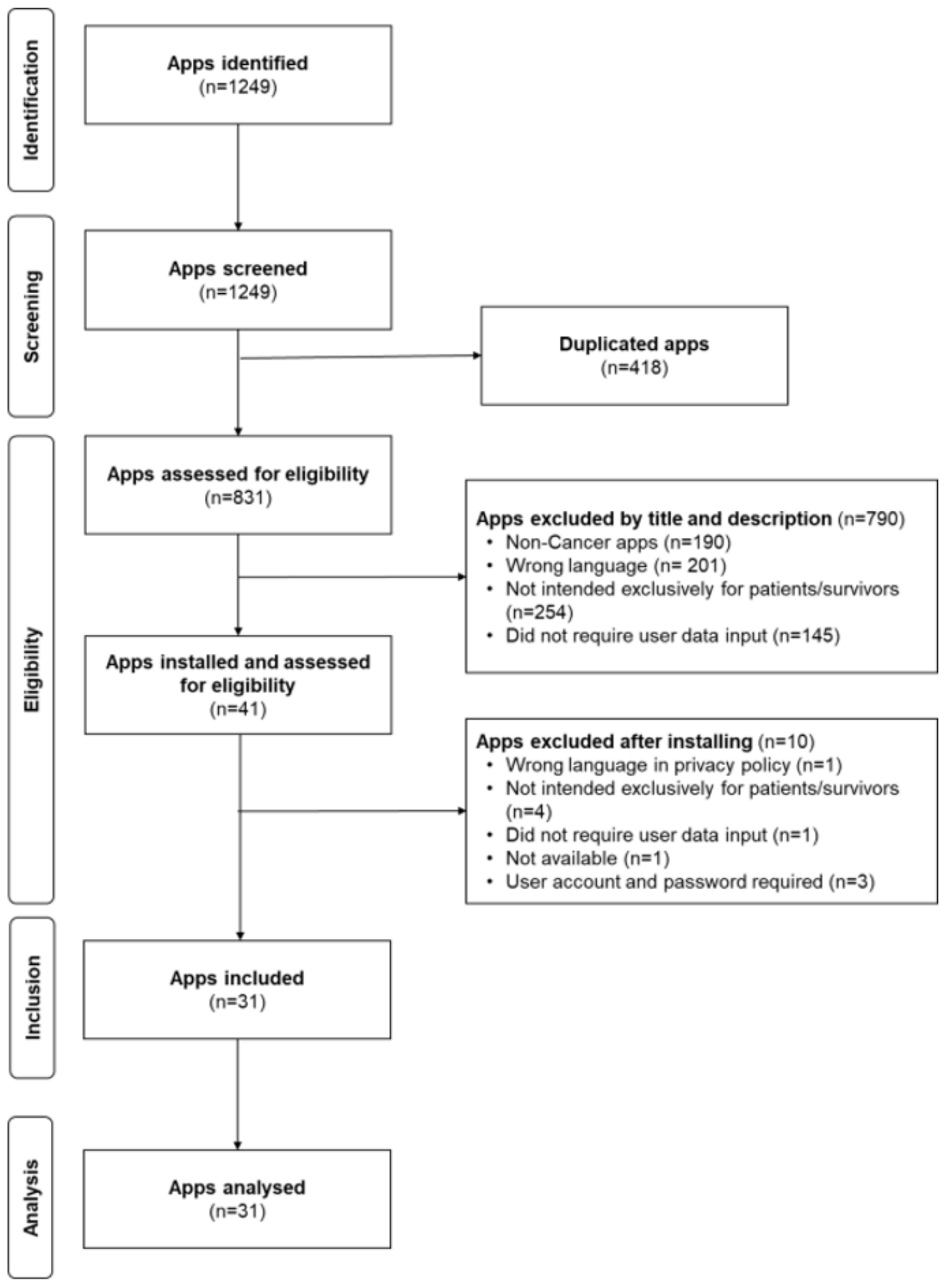


Table 4. Selected apps.

\begin{tabular}{|c|c|c|c|c|c|c|c|}
\hline App name & Developer & $\begin{array}{l}\text { Rating } \\
\text { (stars) }\end{array}$ & \# Ratings & \# Downloads & App type & Cancer type & Label \\
\hline BECCA: Breast Cancer Support & Breast Cancer Care & 4.5 & 63 & $10,000+$ & $\mathrm{S}^{\mathrm{a}}$ & Breast & App1 \\
\hline EmotionSpace cáncer de mama & Pfizer Inc & 2.5 & 2 & $100+$ & $\mathrm{S}$ & Breast & App2 \\
\hline ChemoWave: For Cancer Patients & $\begin{array}{l}\text { Treatment Technologies \& } \\
\text { Insights }\end{array}$ & 4.4 & 20 & $1000+$ & $\mathrm{DM}^{\mathrm{b}}$ & General & App3 \\
\hline OWise Breast Cancer & Px HealthCare BV & 4.4 & 10 & $1000+$ & $\mathrm{DM}$ & Breast & App4 \\
\hline My Cancer Coach & Genomic Health Inc & 4.5 & 86 & $10,000+$ & $\mathrm{DM}$ & General & App5 \\
\hline Breast Advocate & Toliman Health & 5 & 1 & $100+$ & $\mathrm{DTI}^{\mathrm{c}}$ & Breast & App6 \\
\hline Breast Cancer Support & MyHealthTeams & 4.1 & 47 & $1000+$ & $\mathrm{S}$ & Breast & App7 \\
\hline $\mathrm{KMBCN}$ & Kepharge & 5 & 1 & $10+$ & DTI & Breast & App8 \\
\hline Triple Negative Breast Cancer & Kognito & 5 & 2 & $100+$ & DTI & Breast & App9 \\
\hline Breast Cancer: Others Like Me & Eli Malki & 0 & 0 & $5+$ & $\mathrm{S}$ & Breast & App10 \\
\hline Outcomes4Me & Outcomes4Me Inc & 5 & 5 & $100+$ & DTI & Breast & App11 \\
\hline Boobytrapp: The Breast Cancer App & Boobytrapp & 3.7 & 3 & $100+$ & $\mathrm{S}$ & Breast & App12 \\
\hline The BAPS App Wales & $\begin{array}{l}\text { The Orchard Media \& Events } \\
\text { Group Ltd }\end{array}$ & 0 & 0 & $100+$ & $\mathrm{DM}$ & Breast & App13 \\
\hline BELONG Beating Cancer Together & BelongTail & 4.7 & 1,151 & $100,000+$ & $\mathrm{DM}$ & General & App14 \\
\hline Diana & F Hoffmann-La Roche & 5 & 7 & $1000+$ & $\mathrm{DM}$ & Breast & App15 \\
\hline Got Boobs? & Got Boobs & 0 & 0 & $100+$ & $\mathrm{S}$ & Breast & App16 \\
\hline inKind Space & PixelEdge & 0 & 0 & $10+$ & $\mathrm{S}$ & Breast & App17 \\
\hline Adrenal Cancer: Others Like Me & Eli Malki & 5 & 6 & $1000+$ & $\mathrm{S}$ & Other & App20 \\
\hline How Are You Today? PC & Intelesant & 0 & 0 & $100+$ & $\mathrm{DM}$ & Prostate & App21 \\
\hline Cancer.Net Mobile & $\begin{array}{l}\text { American Society of Clinical } \\
\text { Oncology }\end{array}$ & 4.2 & 227 & $10,000+$ & $\mathrm{DM}$ & General & App22 \\
\hline TNM Cancer Staging & $\begin{array}{l}\text { International Atomic Energy } \\
\text { Agency }\end{array}$ & 4.6 & 323 & $10,000+$ & DTI & General & App23 \\
\hline Untire: Beating cancer fatigue & Tired of Cancer BV & 4.5 & 60 & $5000+$ & $\mathrm{DM}$ & General & App24 \\
\hline Self-Care During Cancer & NearSpace Inc & 4.7 & 6 & $1000+$ & $\mathrm{S}$ & General & App25 \\
\hline CanDi: Cancer Diet App & $\begin{array}{l}\text { Faculty of Health Sciences } \\
\text { UniSZA }\end{array}$ & 4.7 & 60 & $500+$ & $\mathrm{DM}$ & General & App26 \\
\hline CancerAid & CancerAid PTY LTD & 3.7 & 25 & $1000+$ & $\mathrm{DM}$ & General & App27 \\
\hline GRYT Health Cancer Community & GRYT Health & 3.9 & 7 & $100+$ & $\mathrm{S}$ & General & App28 \\
\hline $\begin{array}{l}\text { Target Ovarian Cancer Symptoms } \\
\text { Diary }\end{array}$ & Brandwave Marketing & 3.6 & 8 & $1000+$ & $\mathrm{DM}$ & Other & App29 \\
\hline $\begin{array}{l}\text { Pancreatic Cancer Action: Symptom } \\
\text { Tracker }\end{array}$ & Healthbit Ltd & 5 & 3 & $100+$ & $\mathrm{DM}$ & Other & App30 \\
\hline My Care Plan (cancer survivor) & NearSpace Inc & 4 & 4 & $1000+$ & $\mathrm{DM}$ & General & App31 \\
\hline
\end{tabular}

${ }^{\mathrm{a}} \mathrm{S}$ : support.

${ }^{\mathrm{b}} \mathrm{DM}$ : disease management.

${ }^{\mathrm{c}}$ DTI: disease and treatment information. 
Table 5. Privacy scores.

\begin{tabular}{|c|c|c|c|c|c|}
\hline App name & Label & Data controller's location & Last update & $\mathrm{GDPR}^{\mathrm{a}}$ aware & Score \\
\hline BECCA: Breast Cancer Support & App1 & $\mathrm{UK}^{\mathrm{b}}$ & $03 / 2019$ & No & 76.9 \\
\hline EmotionSpace cáncer de mama & App2 & Germany & $05 / 2018$ & No & 75 \\
\hline ChemoWave: For Cancer Patients & App3 & $U^{c}$ & $10 / 2018$ & No & 53.6 \\
\hline OWise Breast Cancer & App4 & $\mathrm{UK}$ & $\mathrm{N} / \mathrm{A}^{\mathrm{d}}$ & Yes & 31.8 \\
\hline My Cancer Coach & App5 & US & $02 / 2015$ & No & 23.1 \\
\hline Breast Advocate & App6 & Unknown & No privacy policy & N/A & 0 \\
\hline Breast Cancer Support & App7 & US & $09 / 2019$ & Yes & 78.6 \\
\hline $\mathrm{KMBCN}$ & App8 & Unknown & No privacy policy & N/A & 0 \\
\hline Triple Negative Breast Cancer & App9 & US & $02 / 2019$ & No & 34.6 \\
\hline Breast Cancer: Others Like Me & App10 & Unknown & No privacy policy & N/A & 0 \\
\hline Outcomes4Me & App11 & Unknown & $11 / 2018$ & No & 34.6 \\
\hline Boobytrapp: The Breast Cancer App & App12 & Singapore & $06 / 2018$ & No & 29.2 \\
\hline The BAPS App Wales & App13 & UK & N/A & Yes & 69.2 \\
\hline BELONG Beating Cancer Together & App14 & Israel & $09 / 2018$ & Yes & 75 \\
\hline Diana & App15 & Spain & $10 / 2018$ & No & 40.9 \\
\hline Got Boobs? & App16 & US & $10 / 2018$ & No & 26.9 \\
\hline inKind Space & App17 & US & N/A & No & 25 \\
\hline Cancer Surveillance & App18 & Unknown & N/A & No & 15 \\
\hline Focalyx & App19 & Unknown & No privacy policy & N/A & 0 \\
\hline Adrenal Cancer: Others Like Me & App20 & Unknown & No privacy policy & N/A & 0 \\
\hline How Are You Today? PC & App21 & Unknown & No privacy policy & N/A & 0 \\
\hline Cancer.Net Mobile & App22 & US & 07/2019 & Yes & 50 \\
\hline TNM Cancer Staging & App23 & Unknown & No privacy policy & $\mathrm{N} / \mathrm{A}$ & 0 \\
\hline Untire: Beating Cancer Fatigue & App24 & Netherlands & N/A & Yes & 66.7 \\
\hline Self-Care During Cancer & App25 & US & $03 / 2014$ & No & 29.2 \\
\hline CanDi: Cancer Diet App & App26 & Unknown & No privacy policy & N/A & 0 \\
\hline CancerAid & App27 & Australia & $\mathrm{N} / \mathrm{A}$ & No & 42.9 \\
\hline GRYT Health Cancer Community & App28 & US & $12 / 2018$ & No & 46.2 \\
\hline Target Ovarian Cancer Symptoms Diary & App29 & $\mathrm{UK}$ & $04 / 2018$ & Yes & 80.8 \\
\hline Pancreatic Cancer Action: Symptom Tracker & App30 & $\mathrm{UK}$ & $06 / 2018$ & Yes & 75 \\
\hline My Care Plan (cancer survivor) & App31 & Unknown & No privacy policy & N/A & 0 \\
\hline
\end{tabular}

${ }^{\mathrm{a} G D P R}$ : General Data Protection Regulation.

${ }^{\mathrm{b}} \mathrm{UK}$ : United Kingdom.

${ }^{\mathrm{c}} \mathrm{US}$ : United States.

$\mathrm{d}_{\mathrm{N}} / \mathrm{A}$ : not applicable.

\section{Assessment of Privacy Policies}

We assessed the fairness of the privacy policies of 31 cancer apps. Surprisingly, as seen in Figure 2A, 29\% (9/31) of the included apps did not have a privacy policy. Thus, we first determined the presence/absence of a privacy policy according to different features in the apps. We considered the app type, the type of cancer, and the number of downloads. A summary of the analysis is shown in Figure 2. Second, we analyzed privacy policies according to the obtained score. As specified in the Methods section, Cat1 (75.0 to 100 points), Cat2 (50.0 to 74.9 points), Cat3 (25.0 to 49.9 points), and Cat4 (0 to 24.9 points). Only $19 \%$ (6/31) of apps had a Cat 1 privacy policy, while 4 apps belonged to Cat 2 . Thus, only $32 \%$ (10/31) of apps scored above 50.0 points in our GDPR-based privacy policy assessment. The results are shown in Figure 3A, where NPP means no privacy policy. We also analyzed the fairness of 
privacy policies according to different features in the apps, considering the app type, type of cancer, and number of downloads. When assessing fairness, we also considered the data controller's country, last privacy policy update, and if the privacy policy was GDPR-aware. A graphic summary of privacy scores can be seen in Figure 3 and Figure 4 .

Privacy policy fairness of 4 included apps had been previously analyzed in Benjumea et al [38]: App1, App4, App7, and App12.
Despite the minor modifications in the scale for the second iteration, App4, App7, and App12 did not change their scores (even though App7 had recently updated its privacy policy). App7's score was one of the highest, with 78.6 points. More remarkable is the case of App1: with a recent update, its score went from 57.7 to 76.9 points. This indicates a significant effort to follow GDPR guidelines.

Figure 2. Analysis of privacy policy presence.

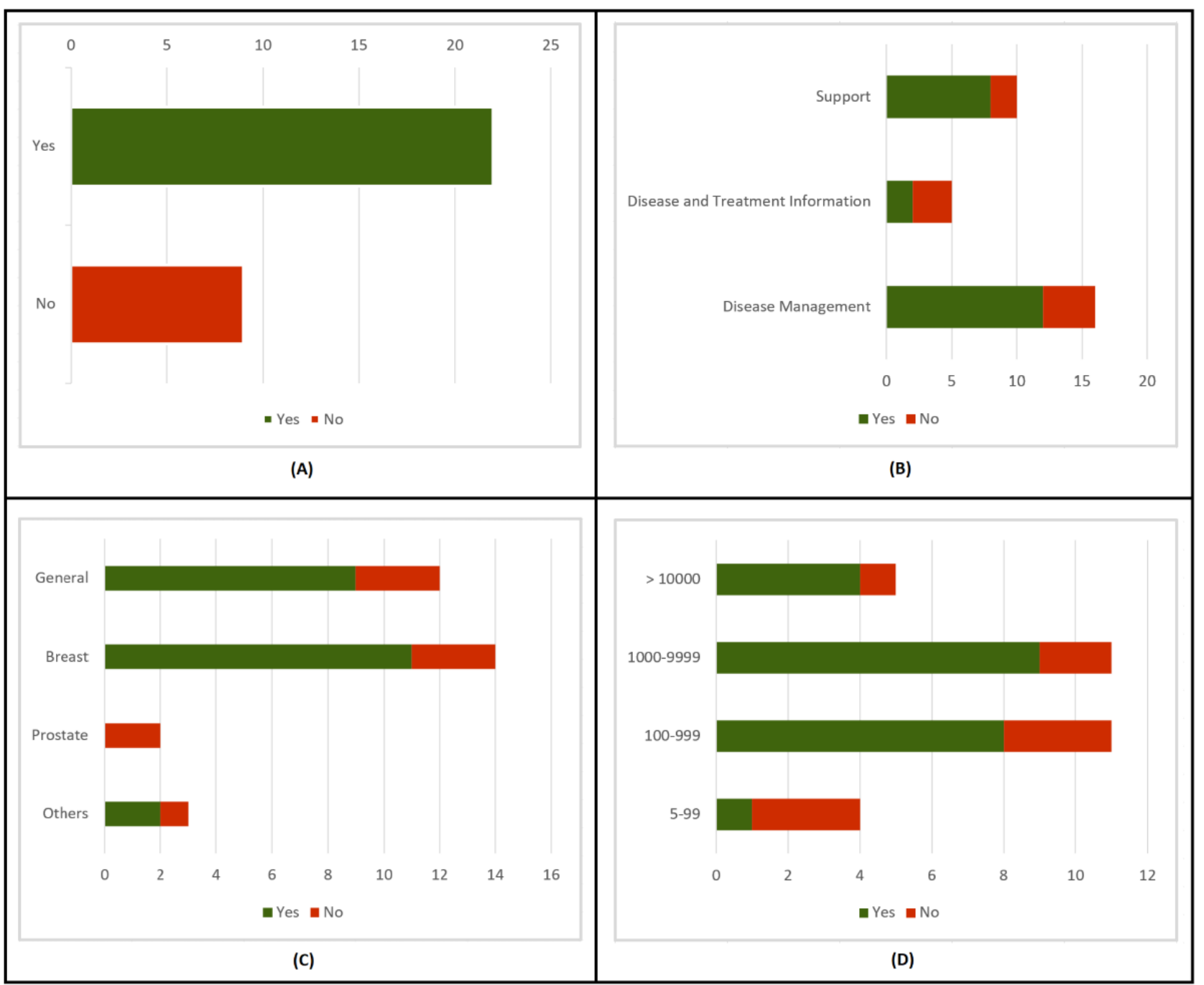


Figure 3. Privacy score summary (part1).

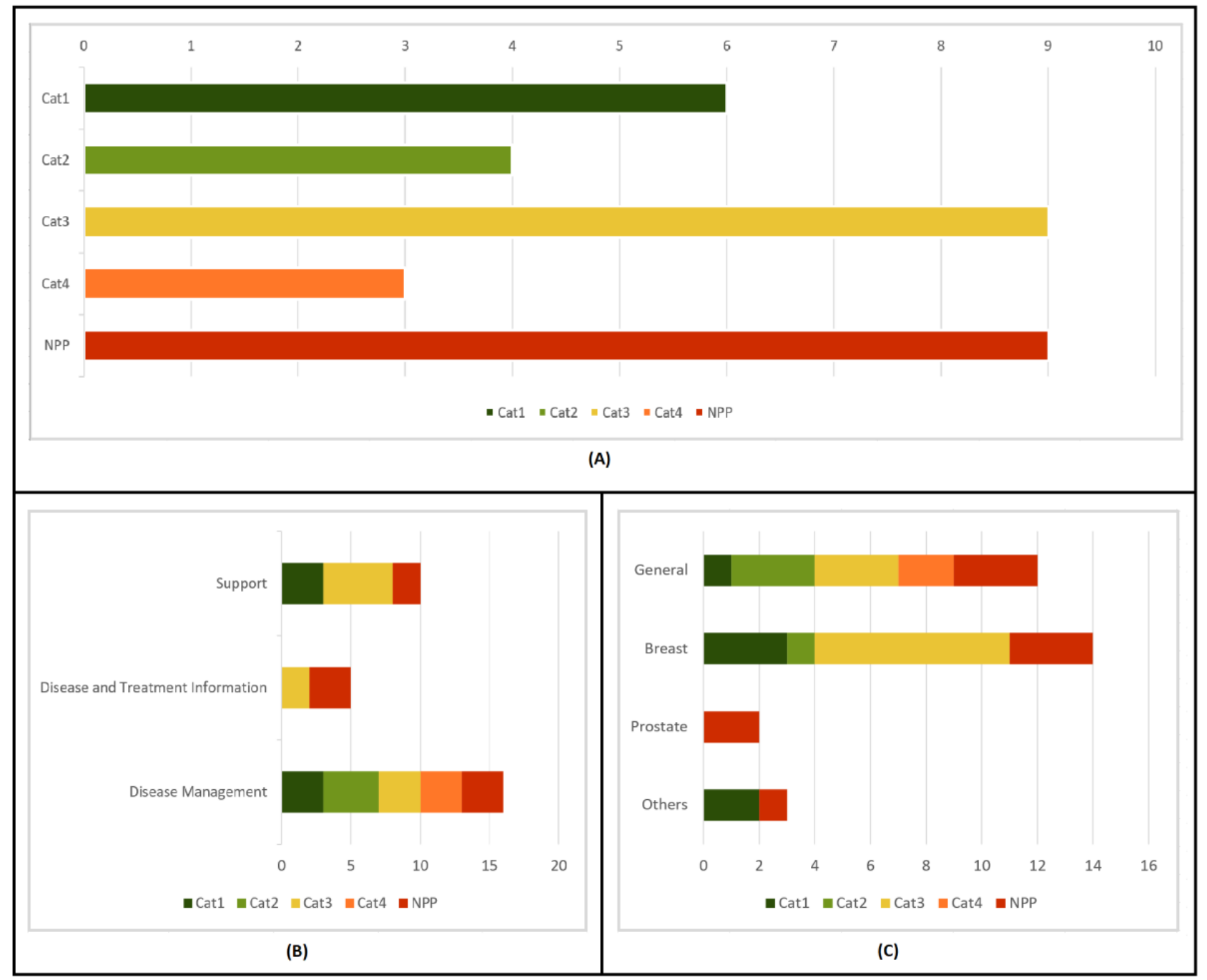


Figure 4. Privacy score summary (part2).

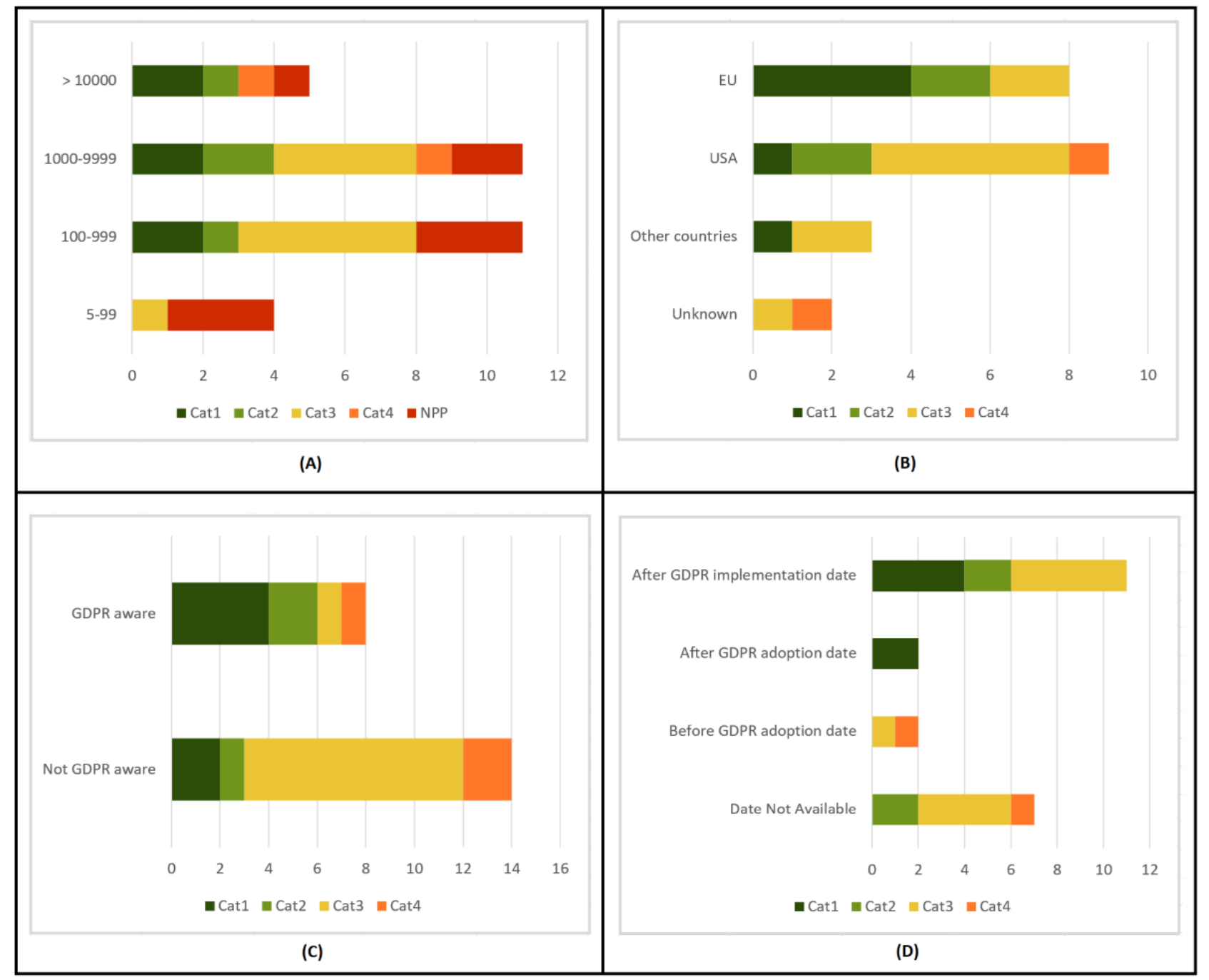

\section{Assessment of Privacy Policies by App Type}

We analyzed the presence of privacy policies according to app type and found that $75 \%(12 / 16)$ of disease management apps and $80 \%(8 / 10)$ of support apps had a privacy policy. As seen in Figure 2B, only $40 \%(2 / 5)$ of disease treatment and information apps had a privacy policy. Regarding the score, $44 \%(7 / 16)$ of disease management apps were above 50 points. Only $30 \%$ (3/10) of support apps were above 50 points, but all of them were in Cat1. No data treatment and information app reached a score of 50. These results are presented in Figure 3B.

\section{Assessment of Privacy Policies by Type of Cancer}

Regarding the presence of a privacy policy according to cancer type, both general cancer apps $(9 / 12,75 \%)$ and breast cancer apps $(11 / 14,79 \%)$ had better results than prostate cancer $(0 / 2)$. However, we consider that only 2 prostate cancer apps are not representative enough for a further analysis. As for other cancer apps, $67 \%$ (2/3) had privacy policies. Results are displayed in Figure $2 \mathrm{C}$. In all, 3 of the breast cancer apps and 1 general cancer app were in Cat1, while the two other type apps that had a privacy policy were also above 75 points. These results do not seem to permit the drawing of any definite conclusions about the relationship between privacy policies and the type of cancer. Results can be seen in Figure 3C.

\section{Assessment of Privacy Policies by Number of Downloads}

Next, we evaluated the relationship between number of downloads and the presence of a privacy policy. We gathered apps into 4 groups: 5 to 99 downloads, 100 to 999 downloads, 1000 to 9999 downloads, and more than 10,000 downloads. The last 3 groups yielded similar results: 80\% (4/5), 82\% (9/11), and $73 \%(8 / 11)$, respectively, had a privacy policy. The only significant difference was observed in apps with 5 to 99 downloads: only $25 \%(1 / 4)$ had a privacy policy. These results can be seen in Figure 2D. There was, however, a difference in scores. None of the apps with 5 to 99 downloads reached 50 points. As for apps with 100 to 999 and 1000 to 9999 downloads, only 27\% (3/11) and 36\% (4/11) of apps, respectively, were in the first two categories. A total of $60 \%$ (3/5) of apps with more than 10,000 downloads were above 50 points. Thus, we found a clear relationship between the number of downloads and the average fairness of privacy policies, which can be observed in Figure 4A.

\section{Assessment of Privacy Policies by Data Controller's Country}

Three more features were analyzed but only in cases where a privacy policy was present. The first was the data controller's country. We identified 4 groups: EU apps (8), US apps (9), apps 
from other countries (3), and apps from unknown countries (2). According to our analysis, the fairness of EU privacy policies was much better than those from the United States, with $75 \%$ (6/8) of EU apps above 50 points, and 4 of them in the first category. Meanwhile, only 33\% (3/9) of US apps were in the first two categories. Only 33\% (1/3) from other countries were above 50 points. As for the two apps for which the data controller's country was unknown, neither reached 50 points. These results can be seen in Figure 4B.

\section{Assessment of Privacy Policies by General Data Protection Regulation Awareness}

The second feature that was analyzed when a privacy policy was present was GDPR awareness. When the GDPR was explicitly mentioned in the app's privacy policy, $75 \%$ (6/8) of those apps reached the first two categories. Moreover, half of them reached the first category. When the GDPR was not mentioned, only $21 \%$ (3/14) reached 50 points. Figure 4C demonstrates this.

\section{Assessment of Privacy Policies by Last Update}

Finally, last privacy policy update was evaluated. We identified 4 groups: apps updated after GDPR implementation (May 25,
2018), apps updated after GDPR adoption (April 14, 2016), apps updated before GDPR adoption, and unknown update date. In fact, half of the 22 apps with a privacy policy were updated after GDPR implementation. Two more were updated after GDPR adoption. We found that $62 \%(8 / 13)$ of the apps updated after GDPR adoption were in the first two categories. Meanwhile, only 22\% (2/9) of the apps not updated after GDPR adoption reached 50 points. Thus, update recency seems to be related to app privacy policy fairness. Figure 4D shows the results.

\section{Assessment of Privacy Policies by Popularity}

Last, we analyzed how popularity affects fairness in privacy policies. Popularity in an app is often measured by the number of downloads and the number of stars [45]. Consistent with previous literature, apps with fewer than 10 ratings were excluded to avoid unfair ratings [46]. Table 6 shows the scores for the apps that have been rated more than 10 times, ordered by their number of stars. Evidently, it is difficult to find any relationship between popularity and privacy score. The apps ranked second and third did not even have a privacy policy, even though App23 had more than 300 ratings. Moreover, App5 was ranked fourth and had a Cat4 privacy score.

Table 6. Assessment of privacy policies by app popularity.

\begin{tabular}{|c|c|c|c|c|}
\hline App label & Stars & Ratings & Downloads & Privacy score \\
\hline App14 & 4.7 & 1151 & $100,000+$ & 75 \\
\hline App26 & 4.7 & 60 & $500+$ & 0 \\
\hline App23 & 4.6 & 323 & $10,000+$ & 0 \\
\hline App5 & 4.5 & 86 & $10,000+$ & 23.1 \\
\hline App1 & 4.5 & 63 & $10,000+$ & 76.9 \\
\hline App24 & 4.5 & 60 & $5000+$ & 66.7 \\
\hline App3 & 4.4 & 20 & $1000+$ & 53.6 \\
\hline App4 & 4.4 & 10 & $1000+$ & 31.8 \\
\hline App22 & 4.2 & 227 & $10,000+$ & 50 \\
\hline App7 & 4.1 & 47 & $1000+$ & 78.6 \\
\hline App27 & 3.7 & 25 & $1000+$ & 42.9 \\
\hline App18 & 3.7 & 21 & $1000+$ & 15 \\
\hline
\end{tabular}

\section{Analysis of Item Compliance}

Finally, we analyzed item compliance for the 22 apps with a privacy policy. Table 7 summarizes the results. We see a heterogeneous compliance of the different items that we checked. Only a few of the items mostly complied. Item 7, showing the recipients of personal data, was satisfied by $95 \%$ (21/22) of apps, while item 4, regarding the purposes of processing, was satisfied by $91 \%$ (20/22) of apps, with the other two giving partial information. Another item with positive results was item 1: $77 \%(17 / 22)$ of apps provided the identity of the data controller, with 3 more apps giving partial information. The last positive item was item 5. A total of $68 \%$ $(15 / 22)$ of apps determined a legal basis for the processing.
Three items showed varied behavior. For item 10, 45\% (10/22) of apps showed the existence of data subject's rights, with 2 more apps giving partial information. For both items 8 and 9 , $36 \%(8 / 22)$ of apps disclosed transfers to other countries and about the period of personal data storage. Some apps gave partial information about them.

A negative behavior was observed for the rest of the items. Only $27 \%(6 / 22)$ of apps satisfied items 3 and 13. Item 3 regarded the DPO's contact details, while item 13 dealt with the obligation of providing personal data and the possible consequences of not providing such data. A total of 6 apps did not comply with item 11, which regarded the right of withdrawing consent at any time. However, item 11 was not applicable in 8 apps, as consent was not a legal basis for data processing. We determined that $23 \%(5 / 22)$ of apps satisfied 
item 12, the right to lodge a complaint with a supervisory authority, with 3 more apps giving partial information. Item 6 is quite particular, as it is only applicable when the legitimate interests of the data controller constitute the legal basis for the processing. Only 33\% (3/9) of apps complied with it, while it was not applicable in 13 apps. Item 14 was satisfied by only
9\% (2/22) of apps. Information about profiling was not available in most of the cases. Last, none of the 13 apps outside the EU complied with item 2 . The apps outside the EU should provide the identity of a representative inside the EU. This item was not applicable to the 9 apps in the EU.

Table 7. Summary of compliance with General Data Protection Regulation items.

\begin{tabular}{|c|c|c|c|c|}
\hline Item number & Full information & Partial information & No information & Not applicable \\
\hline 1 & 17 & 3 & 2 & 0 \\
\hline 2 & 0 & 0 & 13 & 9 \\
\hline 3 & 6 & 0 & 16 & 0 \\
\hline 4 & 20 & 2 & 0 & 0 \\
\hline 5 & 15 & 0 & 7 & 0 \\
\hline 6 & 3 & 0 & 6 & 13 \\
\hline 7 & 21 & 0 & 1 & 0 \\
\hline 8 & 8 & 7 & 6 & 13 \\
\hline 9 & 8 & 5 & 9 & 0 \\
\hline 10 & 10 & 2 & 10 & 0 \\
\hline 11 & 6 & 0 & 8 & 8 \\
\hline 12 & 5 & 3 & 14 & 0 \\
\hline 13 & 6 & 0 & 16 & 0 \\
\hline 14 & 2 & 2 & 18 & 0 \\
\hline
\end{tabular}

\section{Discussion}

\section{Principal Findings}

This paper proposes a GDPR-based scale for assessing the fairness of privacy policies. We defined 14 items that provide developers with a tool to comply with the GDPR, while data controllers and users can use the scale to obtain a score that defines the fairness of privacy policies. Countries are really starting to be concerned about privacy and its implications. This has led the EU to develop laws that help protect user privacy. As a result, the GDPR was adopted in April 2016 and implemented in May 2018. In this study, we developed the second iteration of our GDPR-based method to assess the fairness of privacy policies. In this iteration, we refined the scores and criteria to obtain such scores with the aim of assessing not only compliance with the GDPR but fairness of the privacy policies in an objective way. Discrepancies between researchers have been critically reduced. A percentual score was defined, with a maximum of 100 points. A low score indicated not necessarily that an app did not comply with the GDPR but could indicate a low fairness of its privacy policy.

As privacy is crucial in mHealth and particularly for cancer patients, we assessed 31 Android cancer apps from Google Play. In order to foster straightforward interpretation of the results, we classified scores into four categories according to their fairness (from most to least fair): Cat1, Cat2, Cat3, and Cat4. We analyzed the fairness of privacy policies by app type, cancer type, number of downloads, data controller country, GDPR awareness, and last privacy policy update.
The first disappointing result was the absence of a privacy policy in 9 of 31 apps. This means that only $71 \%$ of the apps had a privacy policy. According to the literature, when top mHealth apps were analyzed, the percentage of apps with a privacy policy was about $90 \%$ [22,23]. However, when the type of app was selected, results were similar to ours: according to Bondaronek et al [32], 75\% of physical activity apps had a privacy policy, while in Adhikari et al [33], they found that $69 \%$ of depression and smoking cessation apps had a privacy policy. When the selection is smaller, results are even worse. O'Loughlin et al [19] found privacy policies in only $49 \%$ of depression apps, while Sunyaev et al [16] showed that, surprisingly, $31 \%$ of medical or health and fitness apps had privacy policies. Moreover, when we analyzed the scores according to the type of app, it was noticeable that disease management apps and support apps obtained better scores than disease treatment and information apps. We believe that this is a positive fact, as disease management apps and support apps handle more sensitive information about patients.

In the literature, there are two ways to assess privacy. Some articles evaluated the different apps according to several items, eventually obtaining a score $[27,28,36]$, while others checked if the analyzed apps met the criteria they had defined [20,32,37].

Regarding scores, only $45 \%$ of apps with a privacy policy that we assessed had a score greater than or equal to $50 \%$, with an average score of 50.5 points. Only Hutton et al [15] had a comparable scoring system. They built a 26 -item heuristic to assess privacy in mHealth apps, although only the first 7 items dealt with privacy policies. They applied their heuristic to 64 
self-tracking mHealth apps and found an average score of $46.2 \%$, with a high dispersion. Thus, their results are quite in line with ours.

The most complied-with items were the following: item 1 (identity of data controller), item 4 (purposes for the processing) and item 7 (recipients or categories of the personal data). Still, only $45 \%$ (5/22) of apps fully informed users about their rights (item 10) and only 5 fully informed users about their right to lodge a complaint with a supervisory authority. Finally, it is interesting that none of the 13 apps whose data controller was not within the EU informed users of the identity of their representative in the EU.

It is difficult to find such a complete analysis in the literature, but some of the items were assessed by different articles. Item 1 was evaluated in Hutton et al [15] and Papageorgiou et al [20], which were complied with by $75 \%$ and $63 \%$ of apps, respectively. Results were similar to our study, where $77 \%$ of apps satisfied this item. Item 1 was also analyzed in Huckvale et al [22], but results were very different. Only $25 \%$ of apps identified the data controller. Item 3 was also evaluated in Papageorgiou et al [20], with none of the apps having a DPO. Our study showed that $27 \%$ of apps had a DPO. Item 4 was assessed in Hutton et al [15] and Minen et al [21]: 61\% and $64 \%$ of apps complied with this item, respectively, whereas a better result $(91 \%)$ was obtained in this paper. Item 7 was assessed in Hutton et al [15], with $61 \%$ of apps stating the recipients of personal data; $96 \%$ of apps makes item 7 the most complied-with item in our study. Item 9 was evaluated in Huckvale et al [22]: $32 \%$ of apps stated the period for which personal data will be stored, compared with $36 \%$. In Minen et al [21], item 10 was analyzed: $36 \%$ of apps informed users about their rights, whereas we obtained a result of $46 \%$. Item 11 was assessed in Hutton et al [15] and Papageorgiou et al [20]: 55\% and $37 \%$ of apps complied with this item, respectively. In our study, $43 \%$ of apps informed users about the right to withdraw consent. Items 12 and 13 were assessed in Huckvale et al [22]: $32 \%$ of apps complied with item 12 , and $36 \%$ of apps satisfied item $13 ; 23 \%$ and $27 \%$ complied with these items in our study. Finally, Papageorgiou et al [20] evaluated item 14: 58\% of apps informed users about profiling. This result was quite different from ours: $9 \%$ satisfied item 14 .

Like other privacy scales $[18,19,27]$, our scale considers each item to be equally important. In further research, we will work on the next iteration of the scale, wherein this approach will be reconsidered. We will evaluate whether using weighted scores provides a better assessment of the privacy policies of mHealth apps or only makes the scale more complex without any additional benefit.

\section{Limitations}

This study has some limitations. Some relevant apps may have been missed during our searches due to limitations of the Google Play search algorithm. Also, it is possible that developers may not have included some relevant information in the app description. As the eligibility assessment was based on app descriptions in the first search, this lack of information might have resulted in app exclusion. Only the Spanish version of the Google Play website was used during the search, and potentially relevant apps published on other versions of Google Play might have been excluded. Our study focused on Android apps, and this restriction also could have introduced a selection bias.

\section{Conclusions}

In this paper, we presented an improved version of our GDPR-based scale for the assessment of the fairness of privacy policies of mHealth apps. This new version has been successfully applied in a case study where the privacy policies of 31 cancer apps were analyzed, yielding results in line with similar studies. This analysis uncovered a surprising lack of fairness in these policies. The nature of the data and the concerns that patients have regarding privacy suggest that it should be a major concern for developers, users, and data controllers. Thus, the proposed scale seems to be suitable for evaluating the fairness of mHealth app privacy policies and for use by developers to ensure compliance with the GDPR.

\section{Acknowledgments}

This work was partially funded by the Cátedra de Telefónica Inteligencia en la red of the Universidad de Sevilla. This work was partially funded by the Cátedra Indra Sociedad Digital of the Universidad de Sevilla. ED-Z receives funding from and is supported by the V Plan Propio de Investigación de la Universidad de Sevilla, Spain.

\section{Authors' Contributions}

JB directed the study and took the lead in defining the scale as well as applying it in the case study. JB also supported the data analysis and interpretation of the data. JR took the lead in drafting the manuscript, supported by JB, OR-R, ED-Z, and AC. JR also participated in study direction, contributed to the scale definition and its application in the case study, and supported the data analysis and interpretation of the data. OR-R took the lead in data collection and participated in study direction, data analysis, and interpretation of the data. ED-Z participated in study direction, data collection, analysis, and interpretation of the data and reviewed the final version of the manuscript. AC resolved discrepancies, acquired funding through a research project, participated in the interpretation of data, and reviewed the final version of the manuscript.

\section{Conflicts of Interest}

None declared. 


\section{Multimedia Appendix 1}

User's guide.

[DOCX File, $71 \mathrm{~KB}-$ Multimedia Appendix 1]

\section{Multimedia Appendix 2}

List of mHealth apps.

[DOCX File, 108 KB-Multimedia Appendix 2]

\section{References}

1. Ham C, Dixon A, Brooke B. Transforming the delivery of health and social care: the case for fundamental change. London:

The King's Fund URL: https://www.kingsfund.org.uk/sites/files/kf/field/field publication file/

transforming-the-delivery-of-health-and-social-care-the-kings-fund-sep-2012.pdf [accessed 2020-07-06]

2. Wyatt K, Finley A, Uribe R, Pallagi P, Willaert B, Ommen S, et al. Patients' experiences and attitudes of using a secure mobile phone app for medical photography: qualitative survey study. J Med Internet Res 2020 May 12;22(5):e14412 [FREE Full text] [doi: 10.2196/14412] [Medline: 32396127]

3. Collado-Borrell R, Escudero-Vilaplana V, Calles A, Garcia-Martin E, Marzal-Alfaro B, Gonzalez-Haba E, et al. Oncology patient interest in the use of new technologies to manage their disease: cross-sectional survey. J Med Internet Res 2018 Oct 23;20(10):e11006 [FREE Full text] [doi: 10.2196/11006] [Medline: 30355554]

4. Bender JL, Yue RYK, To MJ, Deacken L, Jadad AR. A lot of action, but not in the right direction: systematic review and content analysis of smartphone applications for the prevention, detection, and management of cancer. J Med Internet Res 2013;15(12):e287 [FREE Full text] [doi: 10.2196/jmir.2661] [Medline: 24366061]

5. Giunti G, Giunta DH, Guisado-Fernandez E, Bender JL, Fernandez-Luque L. A biopsy of breast cancer mobile applications: state of the practice review. Int J Med Inform 2018 Dec;110:1-9 [FREE Full text] [doi: 10.1016/j.ijmedinf.2017.10.022] [Medline: 29331247]

6. Llorens-Vernet P, Miró J. Standards for mobile health-related apps: systematic review and development of a guide (preprint). JMIR mHealth uHealth 2019. [doi: 10.2196/preprints.13057]

7. Giunti G, Guisado Fernández E, Dorronzoro Zubiete E, Rivera Romero O. Supply and demand in mhealth apps for persons with multiple sclerosis: systematic search in app stores and scoping literature review. JMIR Mhealth Uhealth 2018 May 23;6(5):e10512 [FREE Full text] [doi: 10.2196/10512] [Medline: 29792295]

8. de la Vega R, Miró J. mHealth: a strategic field without a solid scientific soul—a systematic review of pain-related apps. PLoS One 2014 Jul;9(7):e101312 [FREE Full text] [doi: 10.1371/journal.pone.0101312] [Medline: 24999983]

9. Huckvale K, Prieto JT, Tilney M, Benghozi P, Car J. Unaddressed privacy risks in accredited health and wellness apps: a cross-sectional systematic assessment. BMC Med 2015;13:214 [FREE Full text] [doi: 10.1186/s12916-015-0444-y] [Medline: 26404673]

10. Germanakos GP, Mourlas C, Samaras G. A mobile agent approach for ubiquitous and personalized ehealth information systems. 2005. URL: https://cgi.csc.liv.ac.uk/ floriana/UM05-eHealth/Germanakos.pdf [accessed 2020-07-06]

11. Stoyanov SR, Hides L, Kavanagh DJ, Zelenko O, Tjondronegoro D, Mani M. Mobile app rating scale: a new tool for assessing the quality of health mobile apps. JMIR Mhealth Uhealth 2015;3(1):e27 [FREE Full text] [doi: 10.2196/mhealth.3422] [Medline: 25760773]

12. Giunti G, Kool J, Rivera Romero O, Dorronzoro Zubiete E. Exploring the specific needs of persons with multiple sclerosis for mhealth solutions for physical activity: mixed-methods study. JMIR Mhealth Uhealth 2018 Feb 09;6(2):e37 [FREE Full text] [doi: 10.2196/mhealth.8996] [Medline: 29426814]

13. Zhou L, Bao J, Watzlaf V, Parmanto B. Barriers to and facilitators of the use of mobile health apps from a security perspective: mixed-methods study. JMIR Mhealth Uhealth 2019 Apr 16;7(4):e11223 [FREE Full text] [doi: 10.2196/11223] [Medline: $\underline{30990458]}$

14. Regulation (EU) 2016/679 of the European Parliament and of the Council of 27 April 2016 on the protection of natural persons with regard to the processing of personal data and on the free movement of such data, and repealing Directive 95/46/EC (General Data Protection Regulation). URL: https://eur-lex.europa.eu/legal-content/EN/TXT/PDF/ ?uri=CELEX:32016R0679\&from=EN [accessed 2020-07-06]

15. Hutton L, Price BA, Kelly R, McCormick C, Bandara AK, Hatzakis T, et al. Assessing the privacy of mhealth apps for self-tracking: heuristic evaluation approach. JMIR Mhealth Uhealth 2018 Oct 22;6(10):e185 [FREE Full text] [doi: 10.2196/mhealth.9217] [Medline: $\underline{30348623}$ ]

16. Sunyaev A, Dehling T, Taylor PL, Mandl KD. Availability and quality of mobile health app privacy policies. J Am Med Inform Assoc 2014 Aug 21:E28-E33. [doi: 10.1136/amiajnl-2013-002605] [Medline: 25147247]

17. Leigh S, Ouyang J, Mimnagh C. Effective? Engaging? Secure? Applying the ORCHA-24 framework to evaluate apps for chronic insomnia disorder. Evid Based Ment Health 2017 Nov;20(4):e20. [doi: 10.1136/eb-2017-102751] [Medline: 28947676] 
18. Knorr K, Aspinall D, Wolters M. On the privacy, security and safety of blood pressure and diabetes apps. Proc Int Conf ICT Syst Security Privacy Protection 2015. [doi: 10.1007/978-3-319-18467-8 38]

19. O'Loughlin K, Neary M, Adkins EC, Schueller SM. Reviewing the data security and privacy policies of mobile apps for depression. Internet Interv 2019 Mar;15:110-115 [FREE Full text] [doi: 10.1016/j.invent.2018.12.001] [Medline: 30792962]

20. Papageorgiou A, Strigkos M, Politou E, Alepis E, Solanas A, Patsakis C. Security and privacy analysis of mobile health applications: the alarming state of practice. IEEE Access 2018;6:9390-9403. [doi: 10.1109/ACCESS.2018.2799522]

21. Minen MT, Stieglitz EJ, Sciortino R, Torous J. Privacy issues in smartphone applications: an analysis of headache/migraine applications. Headache 2018 Jul;58(7):1014-1027 [FREE Full text] [doi: 10.1111/head.13341] [Medline: 29974470]

22. Huckvale K, Torous J, Larsen ME. Assessment of the data sharing and privacy practices of smartphone apps for depression and smoking cessation. JAMA Netw Open 2019 Apr 05;2(4):e192542 [FREE Full text] [doi: 10.1001/jamanetworkopen.2019.2542] [Medline: $\underline{31002321]}$

23. Scott K, Richards D, Adhikari R. A review and comparative analysis of security risks and safety measures of mobile health apps. AJIS 2015 Nov 22;19. [doi: 10.3127/ajis.v19i0.1210]

24. Brüggemann BT, Hansen J, Dehling T, Sunyaev A. Privacy technologies and policy. In: Schiffner S, Serna J, editors. An Information Privacy Risk Index for mHealth Apps. Cham: Springer; 2016.

25. Mense A, Steger S, Sulek M, Jukic-Sunaric D, Mészáros A. Analyzing privacy risks of mhealth applications. Stud Health Technol Inform 2016;221:41-45. [Medline: 27071873]

26. Zapata B, Hernández Niñirola A, Fernández-Alemán J, Toval A. Assessing the privacy policies in mobile personal health records. Conf Proc IEEE Eng Med Biol Soc 2014;2014:4956-4959. [doi: 10.1109/EMBC.2014.6944736] [Medline: 25571104]

27. Baumel A, Faber K, Mathur N, Kane JM, Muench F. Enlight: a comprehensive quality and therapeutic potential evaluation tool for mobile and web-based ehealth interventions. J Med Internet Res 2017 Mar 21;19(3):e82 [FREE Full text] [doi: 10.2196/jmir.7270] [Medline: 28325712]

28. Bachiri M, Idri A, Fernández-Alemán JL, Toval A. Evaluating the privacy policies of mobile personal health records for pregnancy monitoring. J Med Syst 2018 Jun 29;42(8):1-14. [doi: 10.1007/s10916-018-1002-x] [Medline: 29959535]

29. Robustillo Cortés MDLA, Cantudo-Cuenca MR, Morillo-Verdugo R, Calvo-Cidoncha E. High quantity but limited quality in healthcare applications intended for HIV-infected patients. Telemed J E Health 2014 Aug;20(8):729-735. [doi: 10.1089/tmj.2013.0262] [Medline: 24849001]

30. Quevedo Rodríguez A, Wägner AM. Mobile phone applications for diabetes management: a systematic review. Endocrinol Diabetes Nutr 2019 May;66(5):330-337. [doi: 10.1016/j.endinu.2018.11.005] [Medline: 30745121]

31. Zapata B, Niñirola A, Fernández-Alemán J, Toval A. [Privacy and security in mobile personal health records for Android and iOS]. RISTI 2014 Jun 01(13):35-50. [doi: 10.4304/risti.13.35-50]

32. Bondaronek P, Alkhaldi G, Slee A, Hamilton FL, Murray E. Quality of publicly available physical activity apps: review and content analysis. JMIR Mhealth Uhealth 2018 Mar 21;6(3):e53 [FREE Full text] [doi: 10.2196/mhealth.9069] [Medline: 29563080]

33. Adhikari AR, Richards D, Scott K. Security and privacy issues related to the use of mobile health apps. 2014 Presented at: Proceedings of the 25th Australasian Conference on Information Systems; 2014; Auckland p. 8-10.

34. Aliasgari AM, Black M, Yadav N. Security vulnerabilities in mobile health applications. 2018 Presented at: Proceedings of the IEEE Conference on Applications, Information \& Network Security Nov ; Langkawi, Malaysia; AINS; 2018; Langkawi p. 21-22. [doi: 10.1109/ains.2018.8631464]

35. Mense MA, Urbauer P, Sauermann S, Wahl H. Simulation environment for testing security and privacy of mobile health apps. 2016 Presented at: Modeling and Simulation in Medicine Symposium; 2016; Pasadena p. 3-6. [doi: 10.22360/springsim.2016.msm.008]

36. Powell AC, Singh P, Torous J. The complexity of mental health app privacy policies: a potential barrier to privacy. JMIR Mhealth Uhealth 2018 Jul 30;6(7):e158 [FREE Full text] [doi: 10.2196/mhealth.9871] [Medline: 30061090]

37. Robillard JM, Feng TL, Sporn AB, Lai J, Lo C, Ta M, et al. Availability, readability, and content of privacy policies and terms of agreements of mental health apps. Internet Interv 2019 Sep;17:100243 [FREE Full text] [doi: 10.1016/j.invent.2019.100243] [Medline: 30949436]

38. Benjumea J, Dorronzoro-Zubiete E, Ropero J, Rivera-Romero O, Carrasco A. Privacy in mobile health applications for breast cancer patients. 2019 Presented at: Proceedings of the IEEE 32nd International Symposium on Computer-Based Medical Systems; 2019; Córdoba. [doi: 10.1109/cbms.2019.00131]

39. Informe sobre políticas de privacidad en internet. URL: https://www.aepd.es/media/estudios/ informe-politicas-de-privacidad-adaptacion-RGPD.pdf [accessed 2019-11-01]

40. Decálogo para la adaptación al RGPD de las políticas de privacidad en Internet. URL: https://www.aepd.es/media/estudios/ decalogo-politicas-de-privacidad-adaptacion-RGPD.pdf [accessed 2020-07-06]

41. El deber de informar y otras medidas de responsabilidad proactiva en apps para dispositivos móviles. URL: https://www. aepd.es/media/notas-tecnicas/nota-tecnica-apps-moviles.pdf [accessed 2019-11-01]

42. Fielding R, Reschke J. IETF request for comments. Hypertext transfer protocol (HTTP/1.1): conditional requests. 2014. URL: http://www.ietf.org/rfc/rfc7232.txt [accessed 2020-07-09] 
43. About WHOIS. URL: https://whois.icann.org/en/about-whois [accessed 2019-11-01]

44. Smartphone market share. IDC Corporate. 2020 Jun 22. URL: https://www.idc.com/promo/smartphone-market-share/os [accessed 2019-11-01]

45. Ubaid Ur Rehman M, Aleem M, Islam MA, Ahmed S. Smart applications for diabetes management: a comprehensive survey and ranking. Health Informatics J 2019 Sep 30:1460458219869159. [doi: 10.1177/1460458219869159] [Medline: $\underline{31566463]}$

46. Mojica Ruiz IJ, Nagappan M, Adams B, Berger T, Dienst S, Hassan AE. An examination of the current rating system used in mobile-app stores. IEEE Softw 2016 Nov;33(6):86-92. [doi: 10.1109/ms.2015.56]

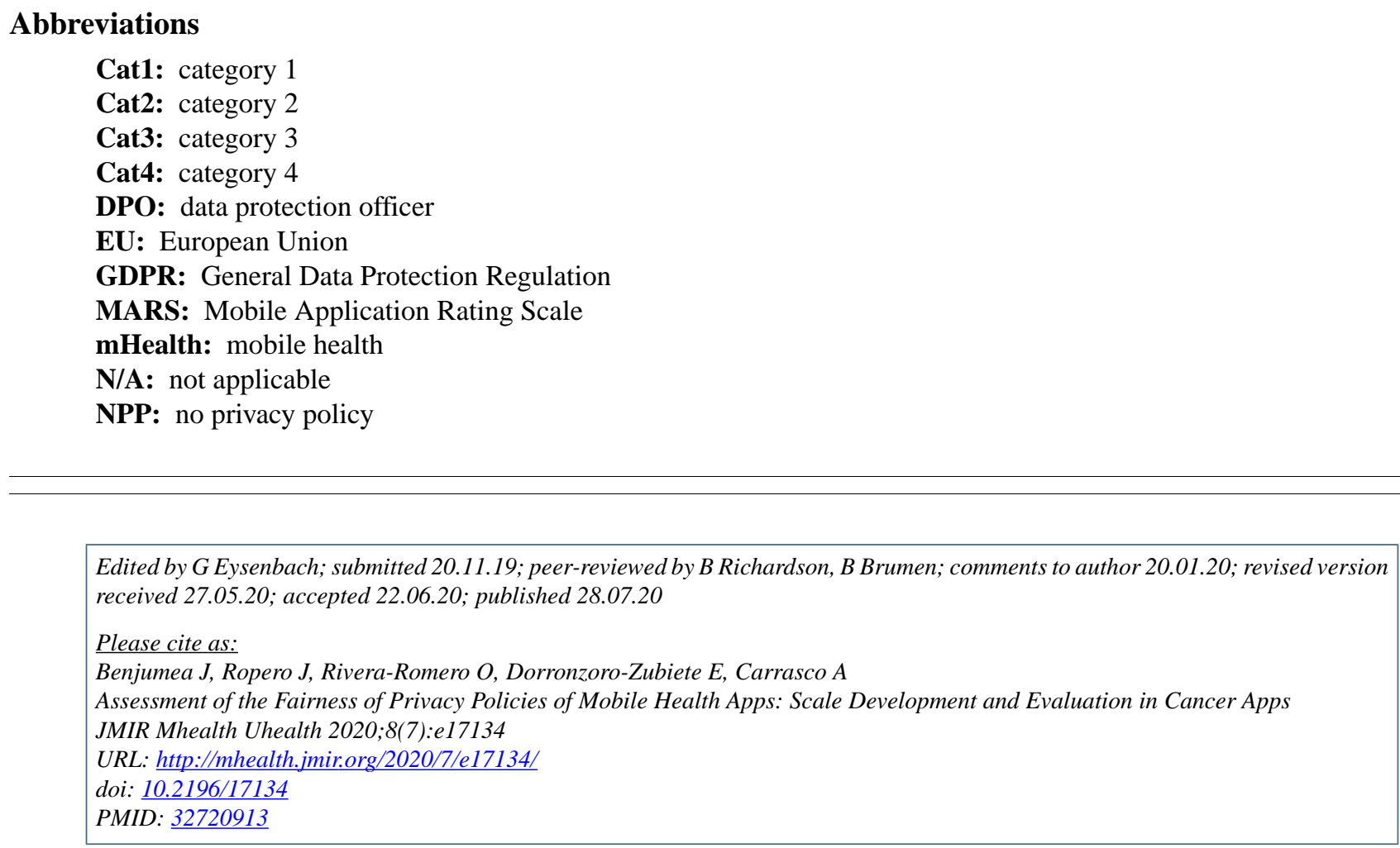

(CJaime Benjumea, Jorge Ropero, Octavio Rivera-Romero, Enrique Dorronzoro-Zubiete, Alejandro Carrasco. Originally published in JMIR mHealth and uHealth (http://mhealth.jmir.org), 28.07.2020. This is an open-access article distributed under the terms of the Creative Commons Attribution License (https://creativecommons.org/licenses/by/4.0/), which permits unrestricted use, distribution, and reproduction in any medium, provided the original work, first published in JMIR mHealth and uHealth, is properly cited. The complete bibliographic information, a link to the original publication on http://mhealth.jmir.org/, as well as this copyright and license information must be included. 